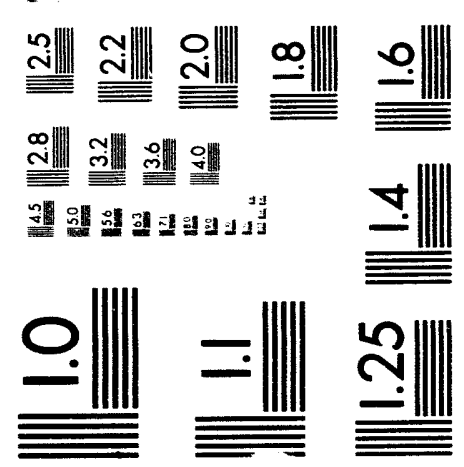



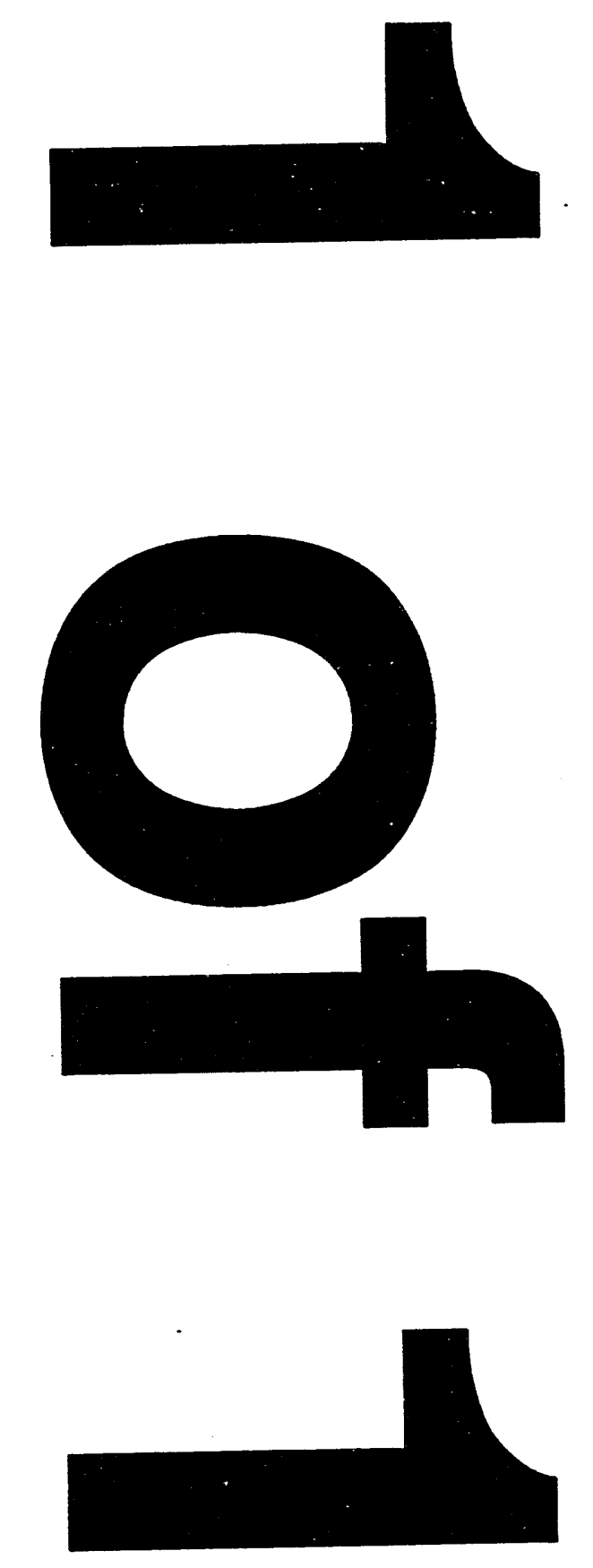


\section{Corf $-9309 / 19-2$}

LA-UR- $93-4482$
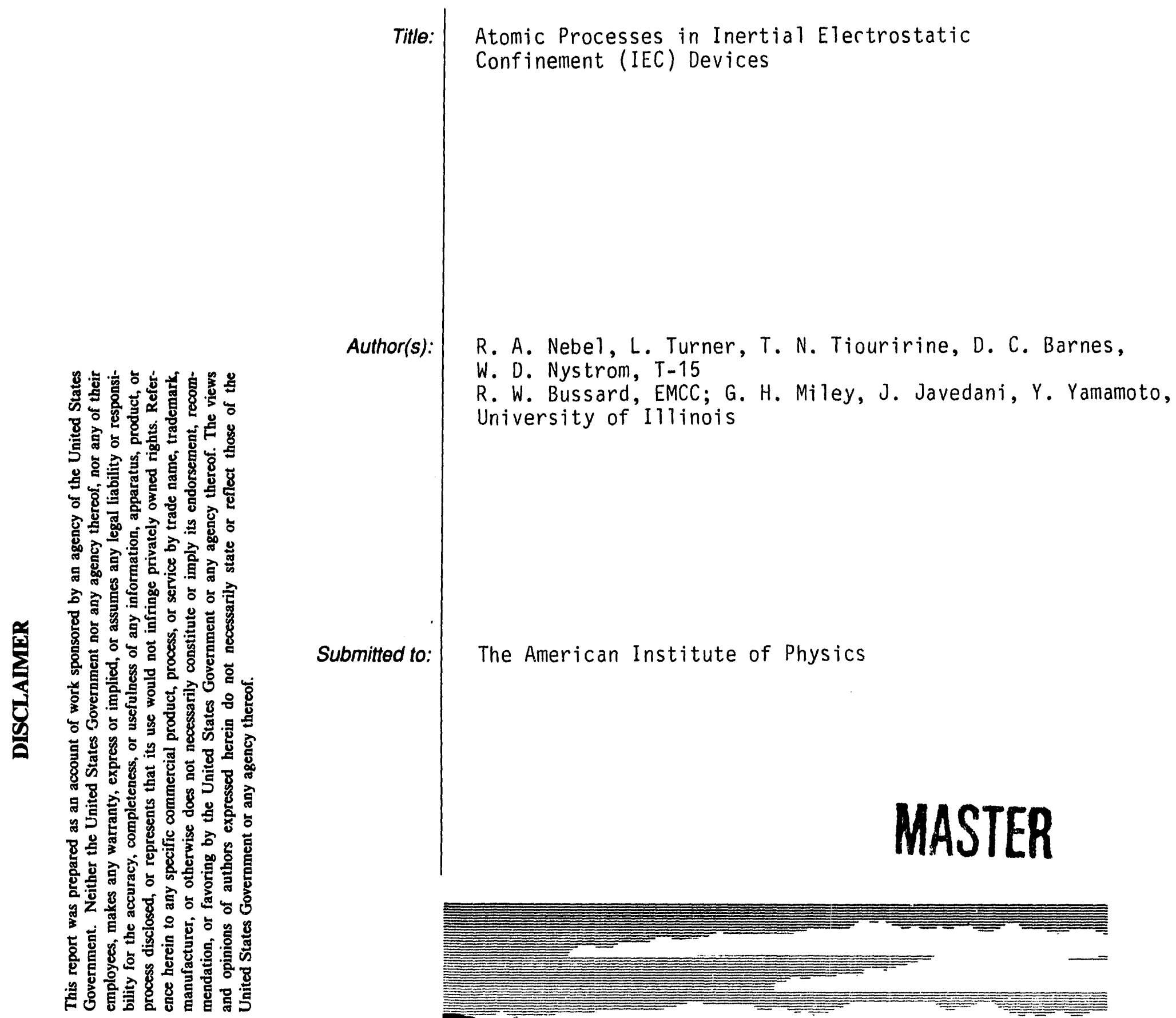

Submitted to:

The American Institute of Physics

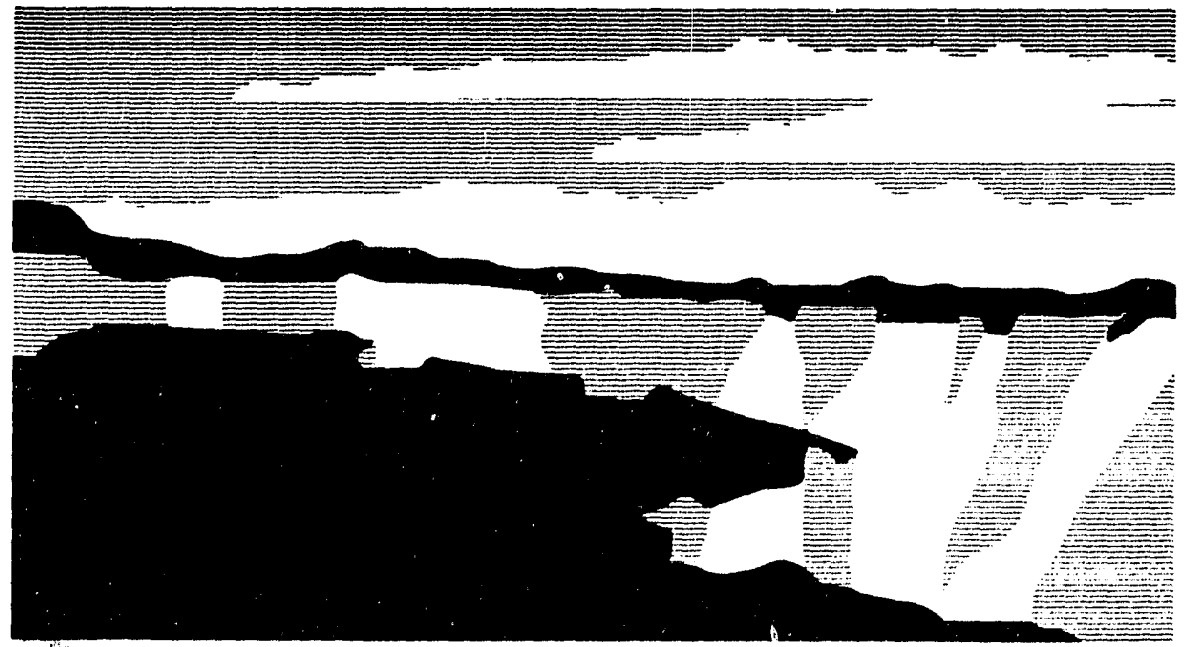

Los Alamos National Laboratory, an affirmative actionequal opportunity employer, is operated by the University of California for the U.S. Department of Energy under contract W-7405-ENG-36. By acceptence of this article, the publisher recognizes that the U.S. Government retains a nonexclusive, royalty-free license to publish or reproduce the published form of this contribution, or to allow others to do so, for U.S. Government purposes. The Los Alamos National Laboratory requests that the publisher identify this anticle as work performed under the auspices of the U.S. Department of Energy. 


\title{
Atomic Processes in Inertial Electrostatic Confinement (IEC) Devices*
}

\author{
R. A. Nebel ${ }^{* *}$, L. Turner, T. N. Tiouririne, D. C. Barnes ${ }^{* *}$, W. D. Nystrom \\ Los Alamos National Laboratory, Los Alamos, NM 87545 \\ R. W. Bussard \\ Energy-Matter Conversion Corporation, Manassas, Va. 22110 \\ G. H. Miley, J. Javedani, Y. Yamamoto \\ University of Illinois, Urbana, Il. 61801
}

\begin{abstract}
Inertial Electrostatic Confinement (IEC) is one of the earliest plasma confinement concepts, having first been suggested by P. T. Farnsworth in the 1950s. The concept involves a simple apparatus of concentric spherical electrostatic grids or a combination of grids and magnetic fields. An electrostatic structure is formed from the confluence of electron or ion beams.

Gridded IEC systems have demonstrated neutron yields as high as $2 * 10^{10}$ neutrons/sec. These systems have considerable potential as small, inexpensive, portable neutron sources for assaying applications. Neutron tomography is also a potential application.

Atomic physics effects strongly influence the performance of all of these systems. Important atomic effects include elastic scattering, ionization, excitation, and charge exchange. This paper discusses how an IEC system is influenced by these effects and how to design around them. Theoretical modeling and experimental results are presented.
\end{abstract}

\section{INTRODUCTION}

While electrostatic confinement has received interest for a number of years, it is only recently that sufficient insight has been gained to realize the important and unique capability of creating a high density fusion source this way. The first suggestion to use electric fields for heating and confining fuel for nuclear fusion reactions was made by Salisbury. 1 Later and better concepts were proposed by Elmore, Tuck and Watson ${ }^{2}$ in 1959, and Farnsworth ${ }^{3}$ in 1956-66. Such concepts have come to be known as inertial-electrostatic confinement (IEC) schemes. They all envision that ions (Salisbury, Farnsworth) or electrons (Elmore, et al.) projected radially inward will confine fusion-reactive ions in a core region. Such confinement has advantages over local thermal equilibrium (LTE) thermonuclear fusion devices in both energy distributior. (monoenergetic vs Maxwellian) and in the absence of collisional loss mechanisms. In the work of Elmore, et al., their original interest was piqued by their theoretical result that a $100 \mathrm{~A}$ electron current could maintain a

\footnotetext{
* work supported by USDOE Advanced Energy Projects and the U. S. Army

* also NambeTech
} 
potential well depth of $100 \mathrm{kV}$ in a device size the order of one meter. However, their analysis, a generalization of the very early work of Langmuir and Blodgett ${ }^{4}$ to two charge species, was limited to radially-Maxwellian plasmas. For these linitited conditions they found that particle densities required to make an economically productive reactor would lead to instabilities.

Furth further explored this configuration in a theoretical study in 1963.5 In this study he concluded that an anisotropic electron distribution in a medium of neutralizing ions could lead to a pinch-type instability possessing a perturbation magnetic field, which could grow if the particle density maintained in the field was too large. Even so, later numerical calculations suggested that net power might be achieved if the density distribution could be kept to at lcast an inverse-square dependence on the radius of the system.

Following this work additional experimental, theoretical and a few computational studies were completed.6-11 Perhaps the most intriguing of these was an experiment done by Hirsch ${ }^{6}$, which indicated that a larger rate of neutron production was occurring than could be accounted for by theoretical and computational models. In this work Hirsch, following the lines conceived by Farnsworth ${ }^{3}$, modified the Elmore et al scheme by using ion beams injected into a sphere to bring in electrons which, in turn, set up the negative potential well structure desired to trap the ions internally.

Hirsch used six symmetrically-placed ion beams of $10 \mathrm{~mA}$ in a background of electrons emitted by a spherical cathode. No instabilities were observed. At least two virtual electrodes were implied (but not measured directly). Reproducible neutron output that increased linearly with current for fixed potential and pressure was produced. A higher neutron yield resilited from lowering the pressure, probably by reduction of beam degradation from neutral-ion and charge exchange reactions at high back pressures.

Hirsch observed $2 * 10^{10} \mathrm{n} / \mathrm{s}$ at densities between $1 * 10^{12}$ and $1 * 10^{14} / \mathrm{cm}^{3}$ with $10 \mathrm{keV}$ and $100 \mathrm{keV}$ ion beams in volumes between $0.1 \mathrm{~cm}^{3}$ and $10 \mathrm{~cm}^{3}$. Hirsch explained these results as being caused by the formation of multiple wells ("poissors") which would enhance the trapping of ions. He found steady-state spherical solutions of Poisson's equation for particles with purely radial motion (i.e. with no transverse momentum) that indicated that such multiple electrostatic wells were conceivable and were consistent with the experimental results. No direct experimental verification was made of his model. Some experimental verification of the existence of potential wells has been made on other experiments, but only for electron-driven electrostatic confinement ${ }^{7-9}$. However, other beam probe experiments have failed to identify a distinctive electrostatic well structure ${ }^{10}$. Indeed, studies ${ }^{8}$ made for ions with finite transverse momentum show the multiple-shell "poissor" structure could not form.

Recently, a series of new exploratory IEC experiments was initiated at the University of Illinois in an effort to verify Hirsch's results. Two spherical IEC devices were constructed, one with ions generated in a gridded configuration via electron-ion collision in an outer region and a second using multiple grids with dispensor cathode electron sources. Results produced thus far with the ion-grid configuration have generally been consistent with Hirsch's data, although the unavailable diagnostics have not allowed exploration of the well structure.

In this paper, we will discuss these experiments, present theoretical models for them, and show how they lead to near term applications as an inexpensive, portable neutron source. We will also show how atomic physics effects play a dominant role in the performance of these devices. 


\section{CONCEPT}

A device of this nature confines a plasma inside a potential well, or a series of multiple potential wells, that is formed from spherical concentric electrodes. The center electrode is charged to a high negative potential, on the order of 10's of kilovolts. Ions will be attracted to this cathode, and if it is highly transparent (on the order of $80 \%$ or higher), this spherical beam of ions will pass through the grid several times before being captured by the electrode. The source of ions could be either ion guns or ionization of background gas, for example. Figure 1 depicts these two types of ion systems. The single grid system operates in "discharge mode," a self sustaining plasma discharge that generates ions; while the three grid system operates in "emitter mode" where electrons flow from emitters about an anode ionizing background gas before their capture by the grid.

\section{RESULTS}

Previous experiments at the University of Illinois have largely operated with a single grid in a discharge mode. There are two principal difficulties with this approach. The first is that breaking down a plasma requires an electron avalanche which necessitates that the plasma be collisional. The fill density for these plasmas is usually several mTorr. The second problem is that the source of electrons for the discharge mode operation is secondary emission from the grid. Ionization occurs as these electrons propagate outward toward the wall. Ionizations occur throughout the entire volume between the grid and the wall. As a result of these two effects, only a small number of ions are accelerated through the entire potential.

Collisions with neutrals rapidly slow down the ions and they tend to accumulate around the grid. This is illustrated in figures $2 a$. and $2 b$. Note the density peak at the grid due to the thermal particles. These calculations were done using the Particle-InCell Monte-Carlo Collision code (PIC-MCC) developed for glow discharges at Berkeley. ${ }^{12}$ These calculations include elastic scattering, ionization, excitation, and charge exchange. Note also that most of the change in the potential interior to the grid occurs near the grid. This is also indicative of there being only a small number of particles with a significant amount of momentum.

Hirsch observed similar behavior in his devices when he operated at high fill pressures. He referred to this type of operation as "hollow cathode mode". 6 However, since Hirsch introduced ions at the boundary via ion guns, he was not restricted to breaking down a background gas in order to produce plasma. He observed that as he lowered the fill pressure, his neutron yields increased.

In our most recent experiments, we have attempted to enter into the low pressure regimes where Hirsch obtained his best results. This is being done using the three grid system shown in figure $1 \mathrm{~b}$. The innermost grid performs the same function as the grid in the single grid system. The outer two grids along with the dispensor cathodes form an effective spherical ion gun. The middle grid is at ground and serves to define the volume of the ion gun and isolate it from the inner grid.

The principles of operation of the ion gun are very simple. The outermost grid is charged to about +500 Volts. Electrons are emitted from the dispenser cathode and are attracted towards the outer grid. They then pass through the grid and continue to oscillate around it in the potential well bounded by the wall and the middle grid (which are both at ground). The purpose of these electrons is to ionize the background gas. The outer grid potential is chosen to be around 500 Volts in order to keep the electron energies near the peak in the ionization cross-sections for hydrogen. Ions which are formed between the outer grid and the middle grid will then be 
accelerated into the interior of the device by the innermost grid. Ions formed between the outer grid and the wall will go to the wall.

Figures $3 a$ and $3 b$ show the results of Particle-In-Cell simulations for the three grid system. The density peak near the innermost grid has now almost disappeared. Note also that the potential has been strongly modified near the origin $(r=0)$ indicating that the particles are carrying significant amounts of momentum.

Typical neutron yields from the three grid experiments are shown in figure 4. Fill pressure for this data was $1-3 * 10^{-4}$ Torr. This data were taken with inner grid currents between $10 \mathrm{~mA}$ and $20 \mathrm{~mA}$. All of the data were taken in deuterium. Hirsch's data indicated there was a conversion factor of about 200 between D-D discharges and D-T discharges. 6 Our best data at higher currents had neutron yields of about $10^{7}$ neutrons/sec in deuterium. This data was at $25 \mathrm{KV}$ while Hirsch's best D-T data $\left(2 * 10^{10}\right.$ neutrons/sec $)$ was achieved at $150 \mathrm{KV} .6$

\section{APPLICATIONS}

Given our present data base, we are confident that we can build a 1010 neutrons/sec source with a power input less than $5 \mathrm{~kW}$. This source will have several attractive features:

1. It's portable.

2. It's inexpensive $(<\$ 100,000)$.

3. It's simple.

4. It's highly reproducible with programmable pulse lengths.

5. It has low radiological risk (total tritium inventory less than $100 \mathrm{mCi}$ ).

6. It produces high energy neutrons (14.7 Mev).

7. It should have a 10000 hour lifetime in a hermetically sealed system.

8. Further optimization of the source may lead to the development of very intense, pulsed, high repetition rate neutron sources.

This is a hot source compared to what is presently commercially available. The present state of the art is the Schlumberger minitron which produces $4 * 10^{8}$ neutrons /sec. (peak) and has a 500 hour lifetime. It's total tritium inventory is a few Ci.

Potential applications for this source include:

1. Nuclear nonproliferation (detection of special nuclear materials).

2. Waste assaying.

3. High explosive detection ("suitcase sniffers").

4. Landmine detection.

5. Drug detection.

6. Well logging.

7. Concrete and coal assaying

A $10^{10}$ neutrons/sec. source is iniense enough that one can erivision using it for neutron tomography applications as well as detection. This would be particularly advantageous in the high explosive and nonproliferation applications. It also opens up the possibility of using the source for applications like detection of pipe cracks in power plants and the like. Since an intense source like this is not presently available, it is likely that a myriad of other applications will arise as well. 


\section{CONCLUSIONS}

Inertial Electrostatic Confinement devices are small, inexpensive plasma devices that appear to have immediate applications as an inexpensive, intense portable neutron source. Since there are more neutrals than plasma in these devices, their physics is dominated by atomic physics effects. Although these devices have been around for some time, very little work has been done to elucidate their physics. We believe this is an exciting area of research which has opportunities for both plasma physicists and atomic physicists.

\section{REFERENCES}

1. W. W. Salisbury, "Method and Apparatus for Producing Neutrons," U. S. Patent No. 2,489,436, issued Nov. 29, 1949, filed Dec. 17, 1947.

2. W. C. Elmore, J. L. Tuck, K. M. Watson, Phys. Fluids 2, 239 (1959).

3. P.T. Farnsworth, "Electric Discharge Devide for Producing Interactions Between Nucleii," U.S Patent No. 3,358,402, issued June 28, 1966, initially filed May 5, 1956, rev. Oct. 18, 1960, filed Jan. 11, 1962.

4. I. Langmuir and K. B. Blodgett, Phys. Rev. 24, 49 (1924).

5. H. P. Furth, Phys. Fluids 6, 48 (1963).

6. R. L. Hirsch, J. Appl. Physics 38, 4522 (1967).

7. T. J. Dolan, J. T. Verdeyen, D. J. Meeker, B. E. Cherrington, J. Appl. Physics 43, 1590 (1972).

8. D. A. Swanson, B. E. Cherrington, J. T. Verdeyen, Appl. Phys. Lett. 23, 125 (1973).

9. D. A. Swanson, B. E. Cherrington, J. T. Verdeyen, Phys. Fluids 16,1939 (1973).

10. W. M. Black, J. W. Robinson, J. Appl. Phys. 53, 2497 (1974).

11. D. C. Baxter, G. W. Siuart, J. Appl. Phys. 53, 4601 (1982).

12. C. K. Birdsall, IEEE Transactions on Plasma Sciences, 19, 65 (1991).

13. D. C. Barnes, et.al., to be published. 


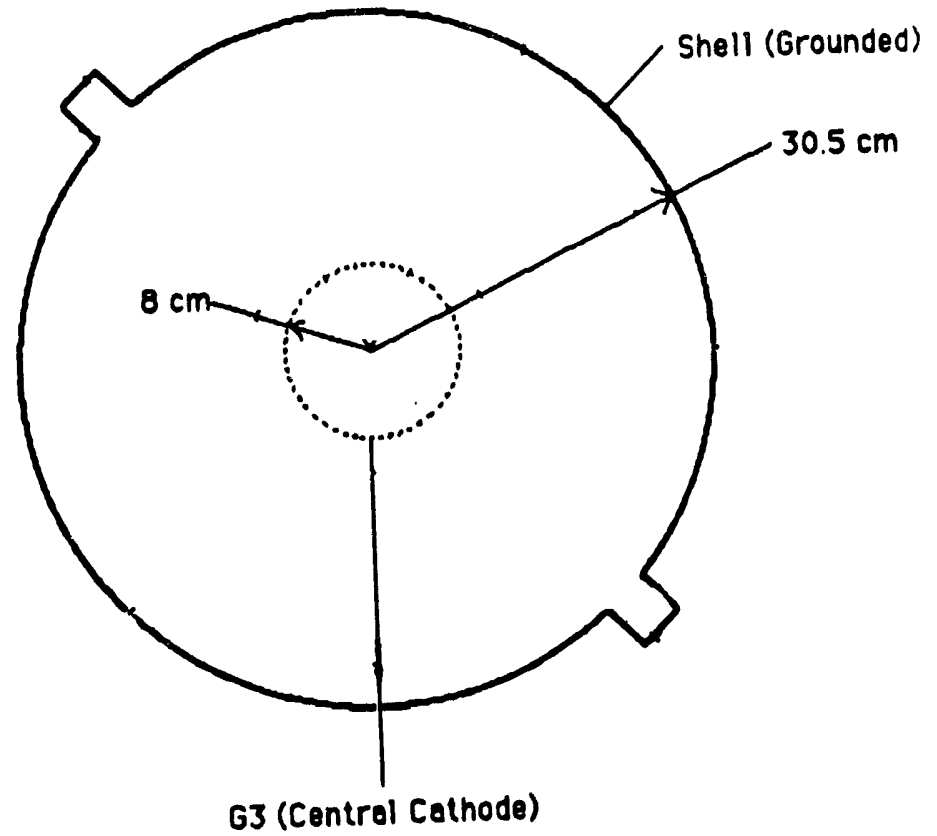

Figure 1a. Single Grid System (Discharge Mode)

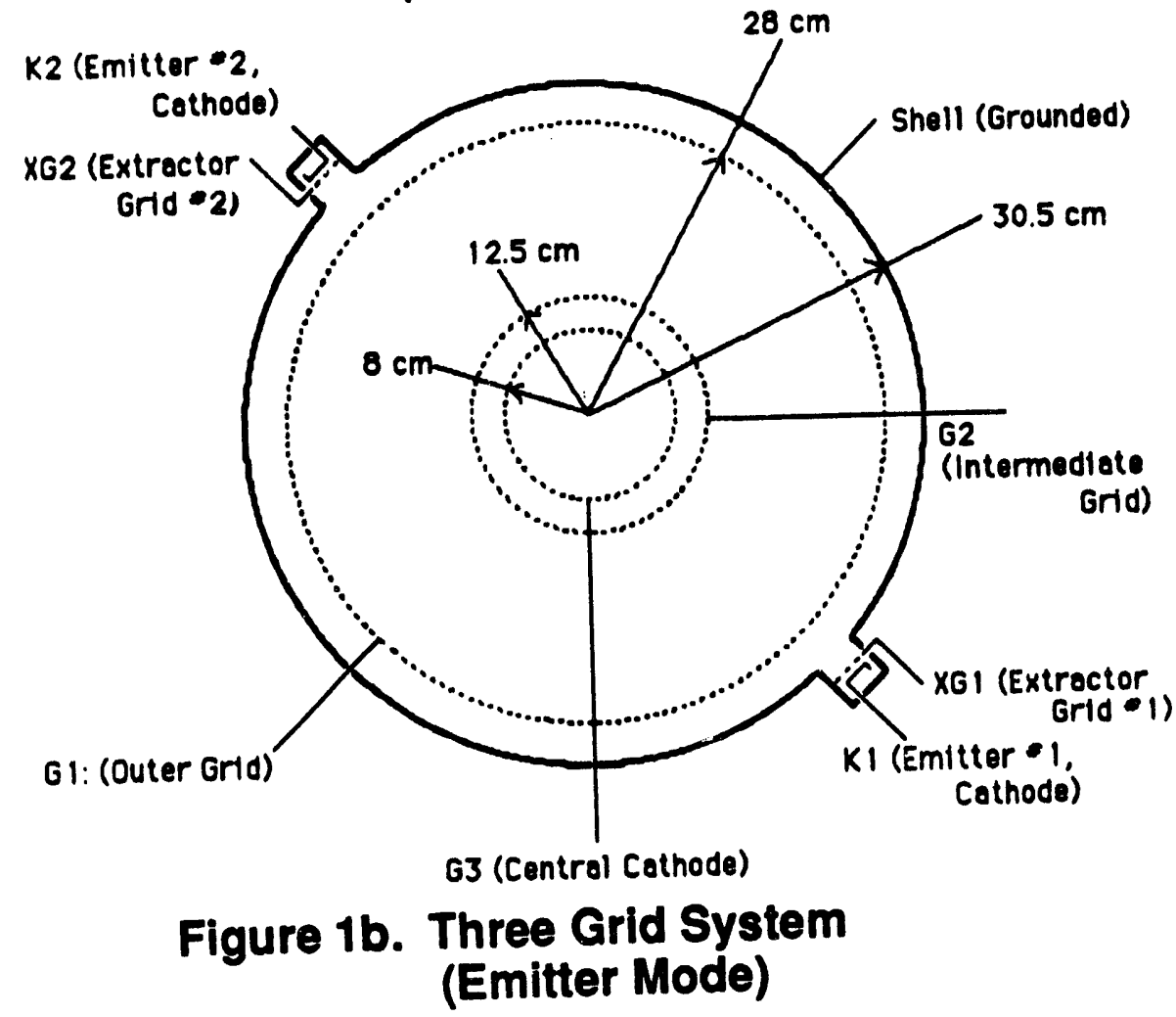




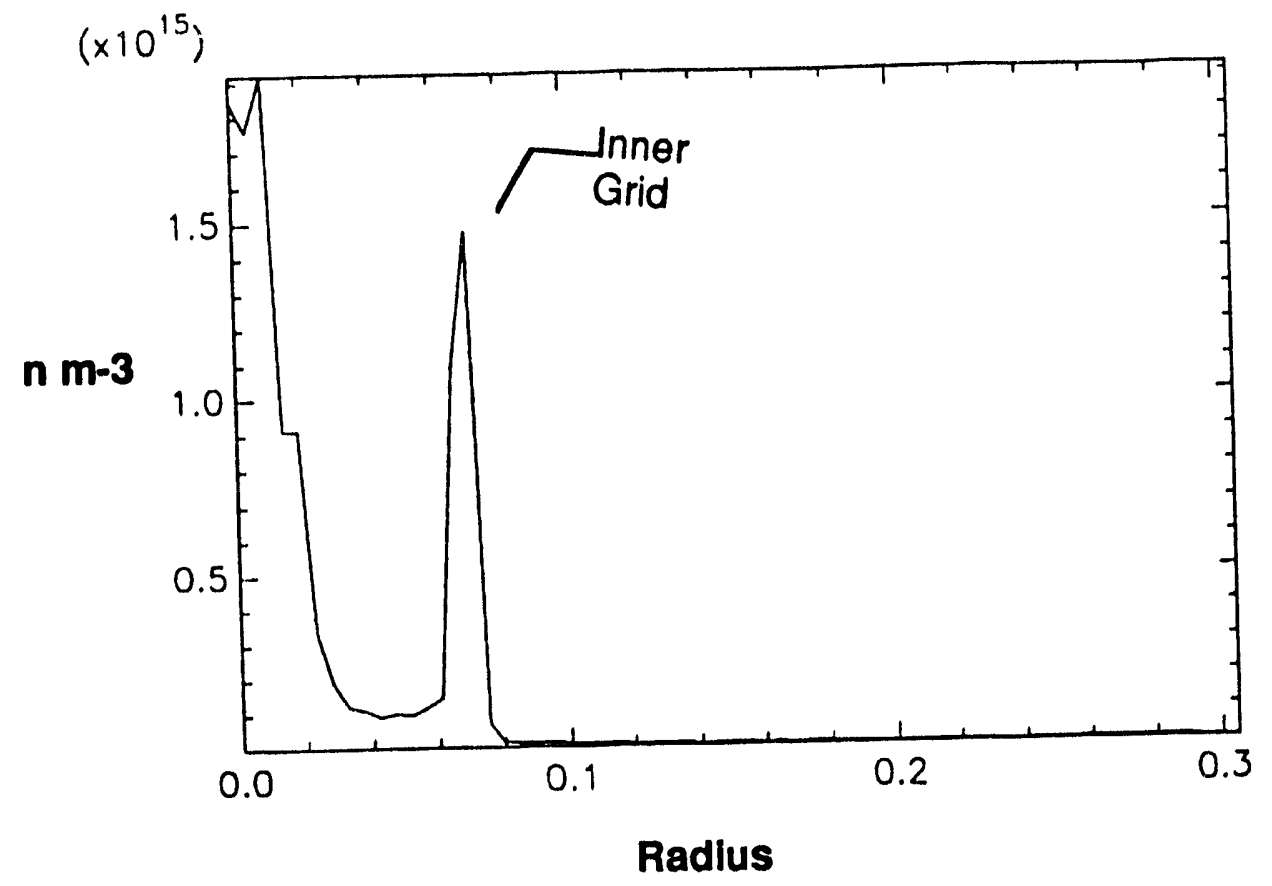

Figure 2a. Ion Density vs. Radius

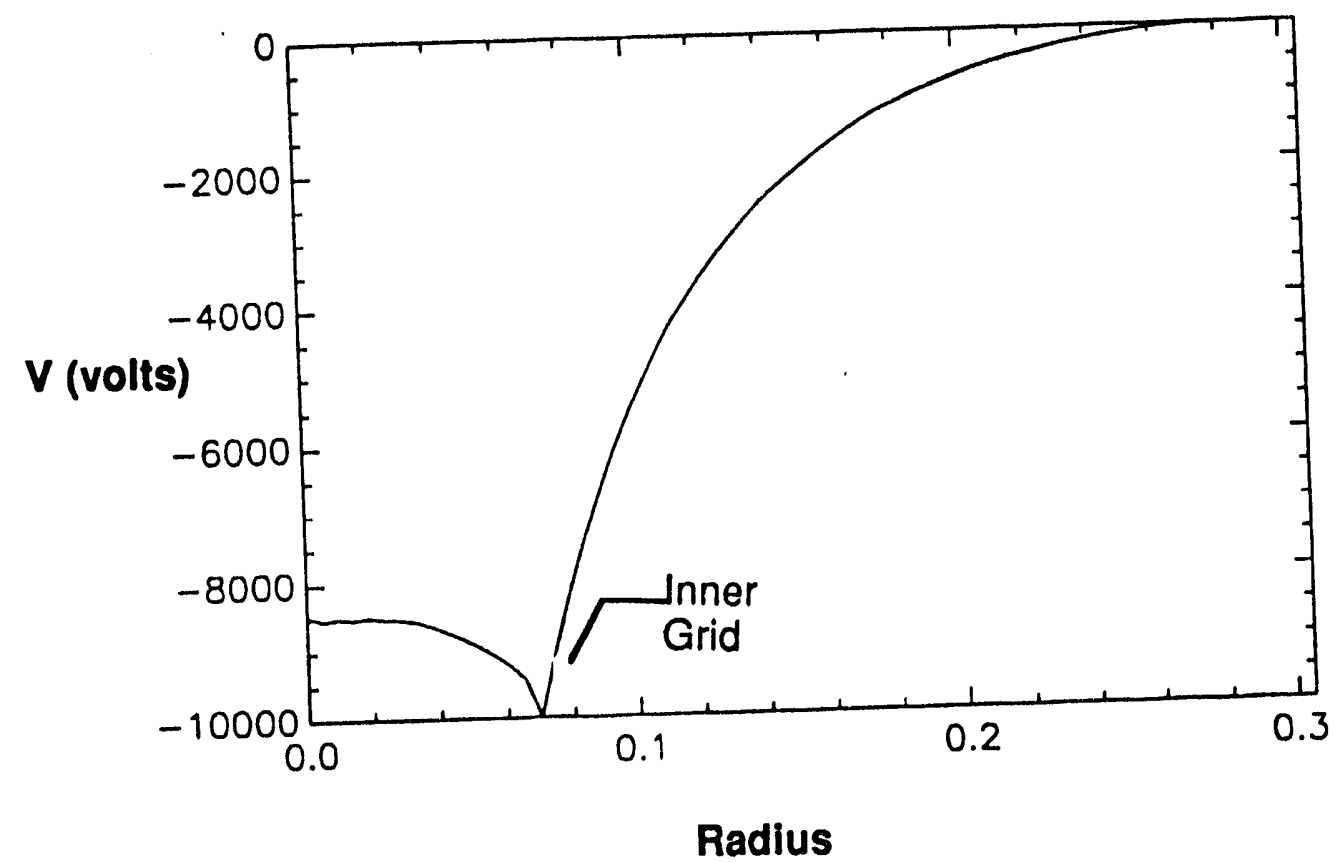

Figure 2b. Electrostatic Potential vs. Radius 


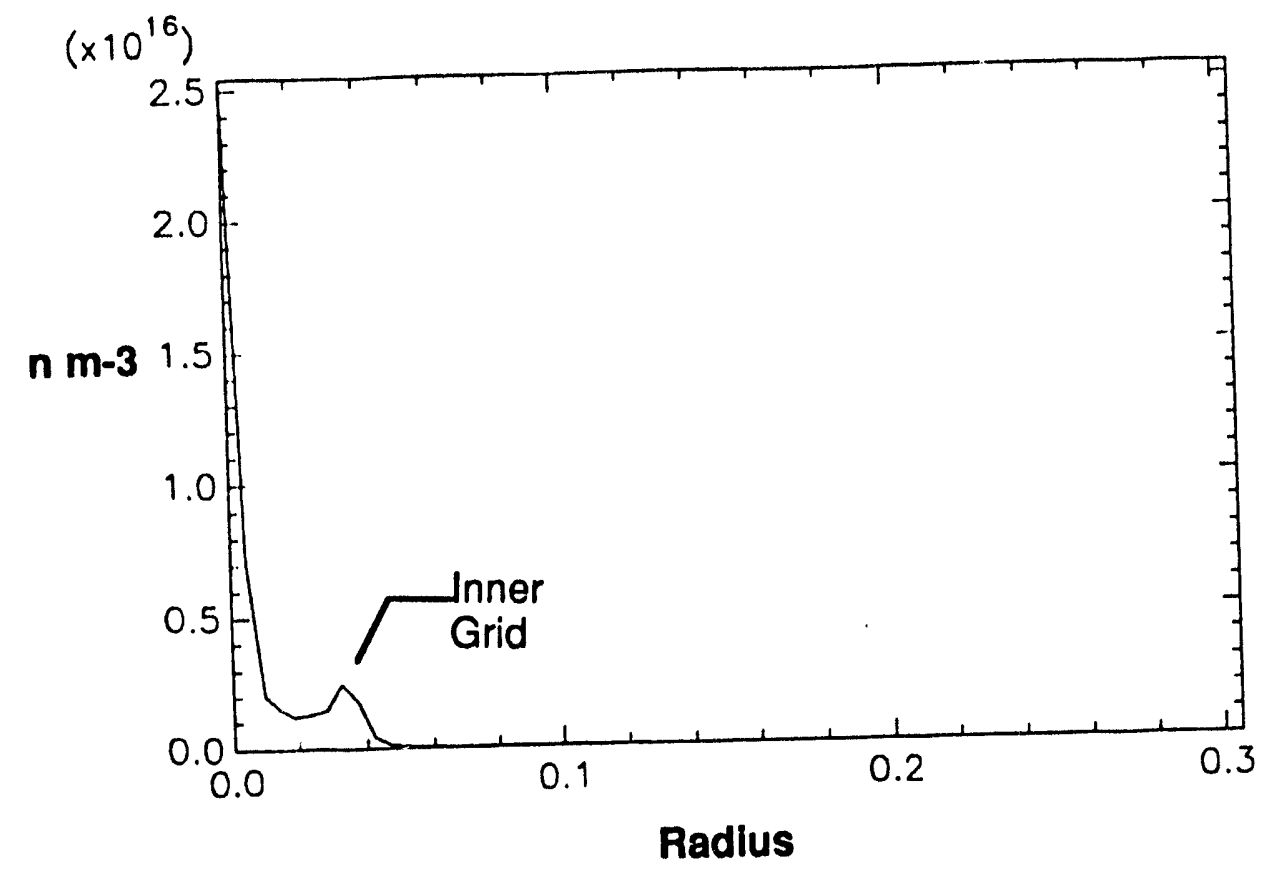

Figure 3a. Ion Density vs. Radius

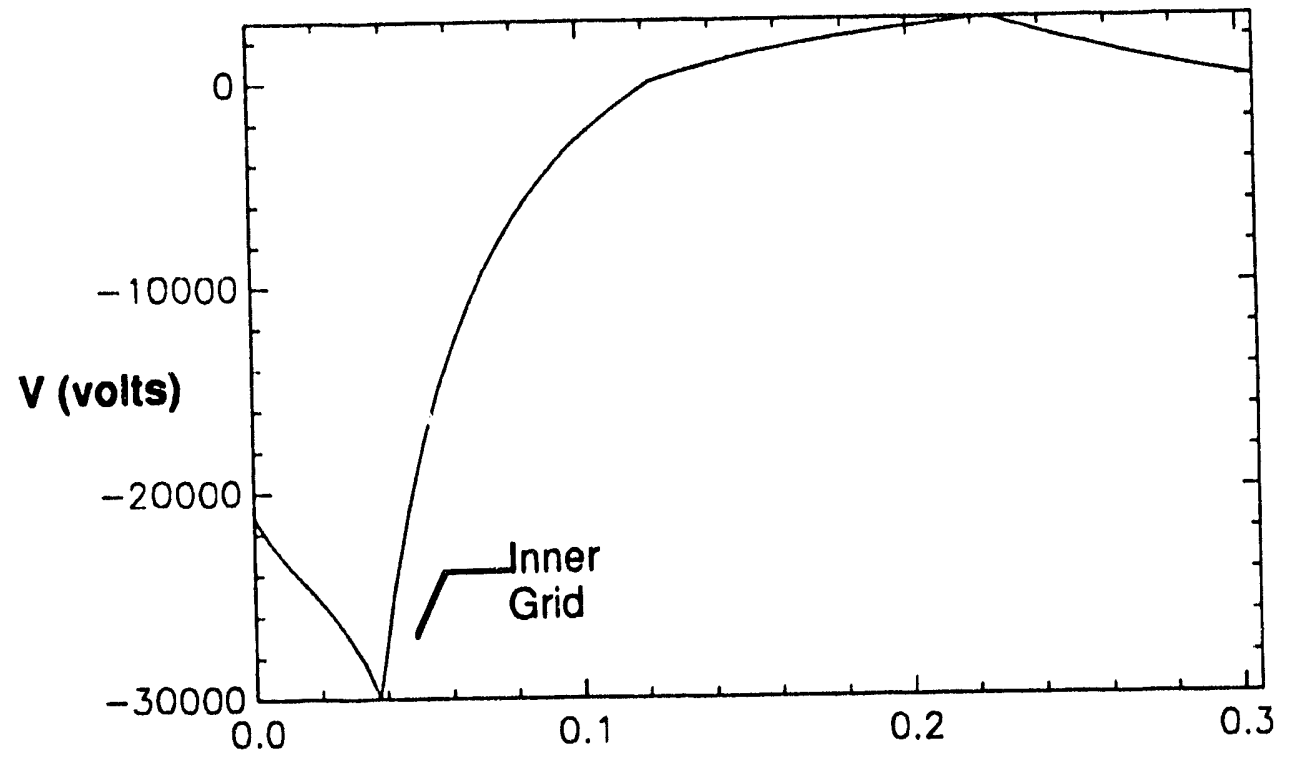

Radius

Figure 3b. Electrostatic Potential vs. Radius 


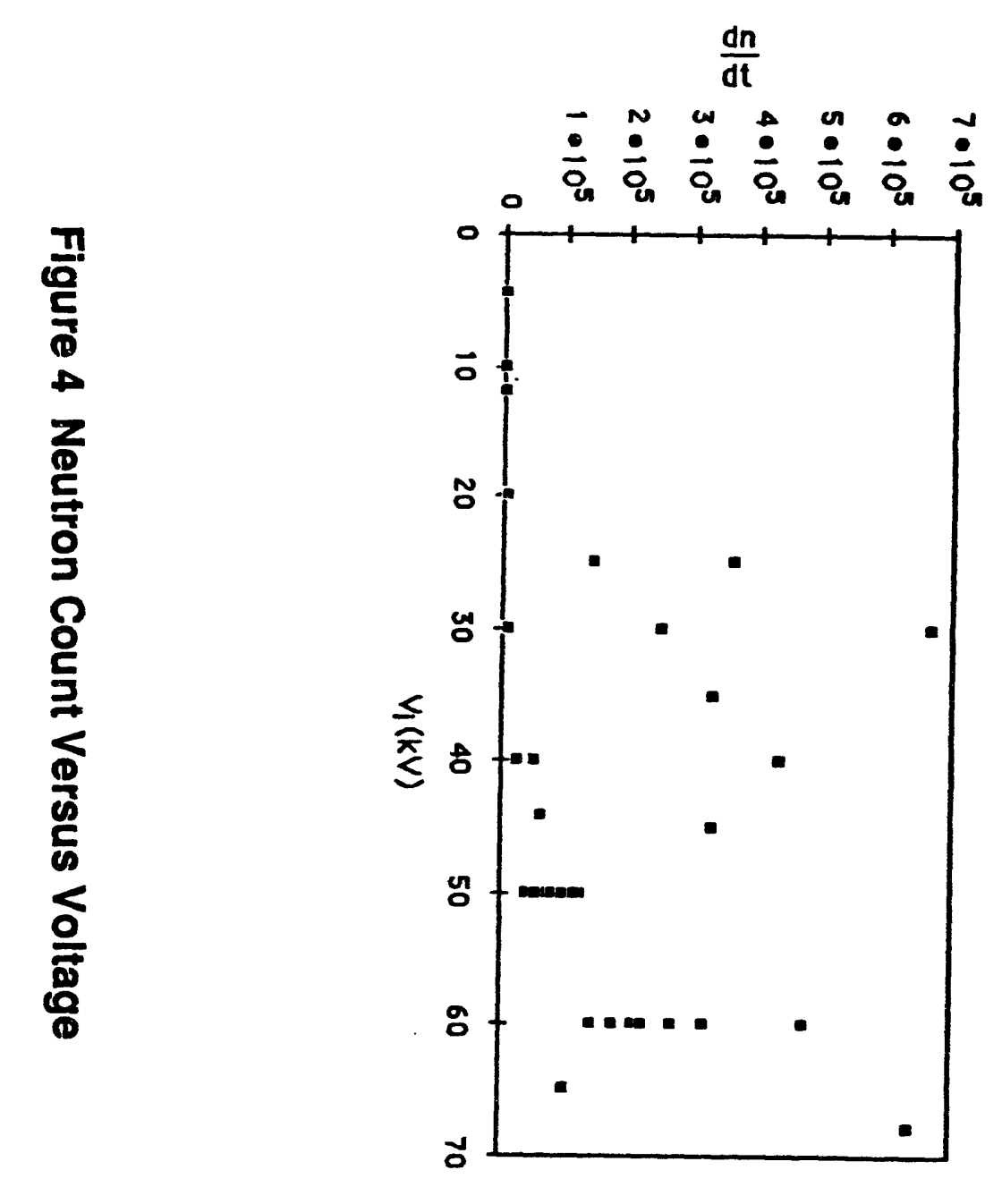


